$69.4 \%$ following the changes. There was no increased 30-day rate of readmission ( $0 \%$ SDD vs $1.3 \%$ all robotic cases) or presentation to the emergency department (1.9\% SDD vs $3.8 \%$ all robotic cases) following implementation of the interventions.

Conclusions Local rates of SDD can be improved with simple interventions targeting disposition planning, foley catheter removal and managing patient expectations. These interventions may be easily applicable to other GO programs.

\section{EPV259/\#288 PATIENT OUTCOMES AND ADHERENCE TO AN ENHANCED RECOVERY PATHWAY FOR OPEN GYNECOLOGIC ONCOLOGY SURGERY: A 5-YEAR SINGLE CENTER EXPERIENCE}

1J Hayek, ${ }^{2} \mathrm{~A}$ Zorrilla-Vaca, ${ }^{3} \mathrm{~L}$ Meyer, ${ }^{2} \mathrm{G}$ Mena, ${ }^{2} \mathrm{~L}$ Lasala, ${ }^{3} \mathrm{M}$ Iniesta, ${ }^{3} \mathrm{~T}$ Suki, ${ }^{4} \mathrm{~K}$ Cain, ${ }^{3} \mathrm{~J}$ Garcia-Lopez, ${ }^{3} \mathrm{P}$ Ramirez. 'Hofstra/Northwell at Staten Island University Hospital, Obstetrics and Gynecology, New York, USA; ${ }^{2}$ The University of Texas MD anderson Cancer Center, Department of Anesthesiology, Houston, USA; ${ }^{3}$ University of Texas MD anderson Cancer Center, Gynecologic Oncology and Reproductive Medicine, Houston, USA; ${ }^{4}$ The University of Texas MD anderson Cancer Center, Division of Pharmacy, Houston, USA

\subsection{6/ijgc-2021-IGCS.330}

Objectives This study evaluates compliance with Enhanced Recovery After Surgery (ERAS) protocol for open gynecologic oncologic surgery at a tertiary center and relationship between levels of compliance and perioperative outcomes.

Methods Our retrospective cohort study included 1879 patients between November 2014 and December 2020. Two groups were identified based on compliance level ( $<80 \%$ versus $\geq 80 \%$ ). Our primary outcomes were 30-day readmission, reoperation, length of stay, and postoperative complications. We also assessed compliance with each ERAS item over time (P1: 2014-2016, P2: 2017-2018, P3: 2019-2020) categorizing patients according to date of surgery. Values were compared between P3 and P1. Multivariable logistic regression analyses were performed to evaluate associations between high compliance and perioperative outcomes.

Results Overall compliance was 74\% (95\%, CI 71.9-78.2). Compliance with ERAS $>80 \%$ was associated with lower Clavien-Dindo grades II (OR 0.74, 95\%CI 0.59-0.93), III (OR 0.55, 95\%CI 0.33-0.93), and V (OR 0.08, 95\%CI 0.010.60 ) complication rates, readmission rates (OR $0.61 ; 95 \% \mathrm{CI}$ $0.43-0.88$ ) and shorter length of stay (OR 0.59; 95\%CI $0.47-0.75)$. Preoperatively, there was increased adherence to counseling $(50 \%, \mathrm{p}=0.01)$, optimization $(21 \%, \mathrm{p}=0.02)$, and carbohydrate-loading $(74 \%, \mathrm{p}=0.02)$. Intraoperatively, use of short-acting anesthetics and adherence to avoiding abdominal drainage $(7 \%, \mathrm{p}=0.04)$ increased. Compliance with goal directed fluid therapy $(16 \%, p=0.04)$ and normothermia $(8 \%$, $\mathrm{p}=0.03)$ decreased. Postoperatively, there was increased compliance with avoiding saltwater overload $(8 \%, \mathrm{p}=0.02)$ and multimodal analgesia $(5 \%, \mathrm{p}=0.02)$.

Conclusions Compliance $(>80 \%)$ with ERAS is associated with lower complication rates, 30-day readmissions, and shorter length of stay without impacting reoperation rates and postoperative opioid use.

\section{EPV260/\#451 SCHWANNOMA OF THE PUDENDAL NERVE - ANATOMICAL CONSIDERATIONS IN THE APPROACH TO SURGICAL MANAGEMENT OF THIS RARE PATHOLOGY}

${ }^{1} S$ Smyth*, ${ }^{2} \mathrm{~L}$ Cogswell, ${ }^{3}$ S Dhar, ${ }^{1} \mathrm{H}$ Soleymani Majd. ${ }^{1}$ Oxford University Hospitals NHS Foundation Trust, Gynaecological Oncology, Oxford, UK; ${ }^{2}$ Oxford University Hospitals NHS Foundation Trust, Plastic Surgery, Oxford, UK; ${ }^{3}$ Oxford University Hospitals NHS Foundation Trust, Histopathology, Oxford, UK

\subsection{6/ijgc-2021-IGCS.331}

Objectives Schwannomas are uncommon, benign, indolent, nerve sheath tumours with low malignant potential; commonly affecting the nerves of the head, neck, mediastinum and extremities. Two pudendal cases have been reported. Their deep, complex location challenges resection - via the ischiorectal fossa in our case.

Methods A 24-year-old female reported an enlarging left perineal mass over eight years; associated with numbness and sexual dysfunction. This was $7 \times 5 \mathrm{~cm}$ on examination, distal to Alcock's canal, with no vaginal, rectal or anal sphincter involvement. It was defined and mildly FDG-avid on imaging. Biopsies confirmed schwannoma. She reports no neurological deficit or evidence of recurrence following resection.

Results Alcock's canal is directly accessed via the ischiorectal fossa with minimal pelvic muscle and ligament disruption. The pudendal nerve arises from the S2-4 sacral nerve and travels forward laterally in the pelvis within this obturator internus fascial sheath. It has both motor and sensory functions. The ischiorectal fossa is a pyramidal space lateral to the anal canal and below the pelvic diaphragm. It contains the internal pudendal and inferior rectal vessels and nerves.

Conclusions Pudendal nerve schwannomas are rare, arising from a single non-functioning sensory fascicle. Following

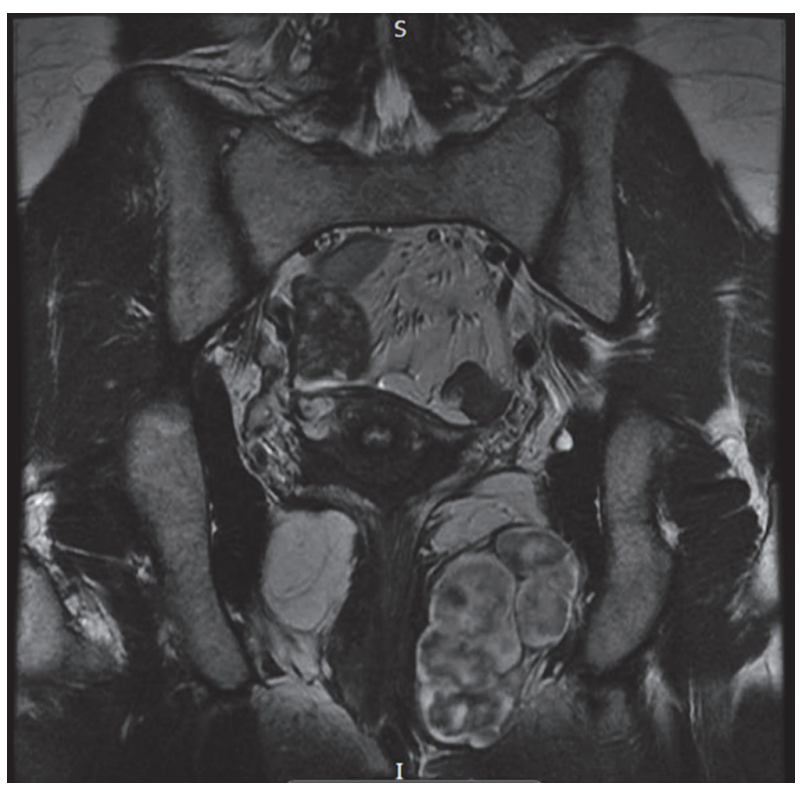

Abstract EPV260/\#451 Figure 1 


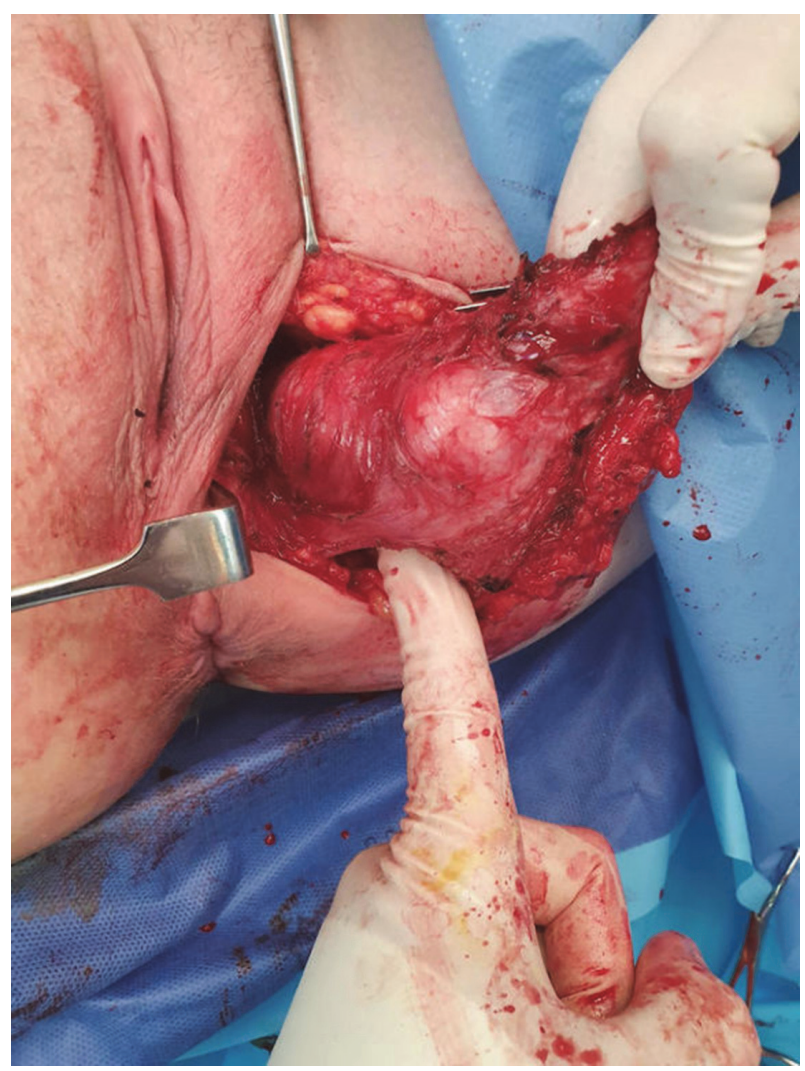

Abstract EPV260/\#451 Figure 2

preoperative imaging, this approach is safe and effective to achieve complete surgical resection, avoiding relapse. Other risk factors include incontinence and sensory defecit. The procedure requires an in depth knowledge of the pelvic anatomical spaces, their contents and boundaries.

\section{EPV261/\#46 TOTAL LAPAROSCOPIC HYSTERECTOMY IN MORBIDLY OBESE PATIENTS - REDUCING THE RISK}

E Van Der Zanden, F Testa, C White, S Kaushik, P Larsen-Disney, F Drews, S Baron* Brighton and Sussex University Hospital NHS Trust, Gynaecological Oncology, Brighton, UK

\subsection{6/ijgc-2021-IGCS.332}

Objectives To evaluate whether a consultant 'buddy' operating approach improves on intra-operative and post-operative outcomes in patients undergoing total laparoscopic hysterectomy (TLH) for endometrial cancer who are extremely and morbidly obese.

Methods A prospectively selected cohort of 25 patients with a BMI 47-70 undergoing TLH was divided into two groups according to whether the first assistant to the Gynae-Oncology consultant was a registrar, or a consultant ('buddy operating'). Anaesthetic time, operating time, intraoperative estimated blood loss (EBL), requirement for high dependency unit (HDU) bed and length of stay (LOS) were compared.

Results Average 'buddy' operating time was significantly shorter compared to the registrar-assistant group $(01: 31 \mathrm{~h}$ vs $01: 59 \mathrm{~h}$ respectively; $\mathrm{p}<0.001)$; a similar trend was seen with the average total anaesthetic time $(02: 48 \mathrm{~h}$ vs $03: 23 \mathrm{~h}$ respectively; $\mathrm{p}<0.001)$. EBL was less in the 'buddy operating' group (39 mls) vs registrar-assistant group (169 mls; $\mathrm{p}<0.001)$. Post- operatively, LOS was shorter in the 'buddy operating' group as compared to the registrar-assistant, though not significantly so $(1.13$ vs 1.59 days; $p=0.109) .2$ of the total patients $(8 \%)$ required a one-night stay in HDU for observation due to their co-morbidities, both in the registrar-assistant group. Mean BMI, age, ASA and comorbidities were similar in the two groups.

Conclusions In patients with a significantly raised BMI, TLHs by two consultants vs consultant and registrar are associated with better intra and post-operative outcomes, including reduced overall anaesthetic time, operating time, and EBL. There is an association with a reduced length of overall hospital stay, though this was not significant.

\section{EPV262/\#462 FUNCTIONAL NEOVAGINA FORMATION USING VERTICAL RECTUS ABDOMINIS MUSCULOCUTANEOUS (VRAM) FLAP FOLLOWING PELVIC EXENTERATION}

${ }^{1} \mathrm{~S}$ Smyth*, ${ }^{1} \mathrm{C}$ Pappa, ${ }^{1} \mathrm{H}$ Jiang, ${ }^{2} \mathrm{R}$ Mykula, ${ }^{1} \mathrm{M}$ Alazzam. ${ }^{1}$ Oxford University Hospitals NHS Foundation Trust, Gynaecological Oncology, Oxford, UK; ${ }^{2}$ Oxford University Hospitals NHS Foundation Trust, Plastic Surgery, Oxford, UK

\subsection{6/ijgc-2021-IGCS.333}

Objectives Cervical cancer is common in younger women, with risks of surgery for advanced disease including altered body image and psychosexual dysfunction. This specialist technique highlights multidisciplinary management whilst minimising complications.

Methods A 42-year-old female with a stage 1B2 lymph node positive cervical adenocarcinoma managed with primary chemoradiotherapy underwent total pelvic infralevator exenteration for recurrence; with vulval sparing and VRAM reconstruction of the pelvic floor, perineum and neovagina formation.

Results Following preoperative marking and perforating vessel Doppler identification of the anterior abdominal wall; the anterior rectus sheath was preserved to the medial row and exenteration procedure completed with staggered urostomy

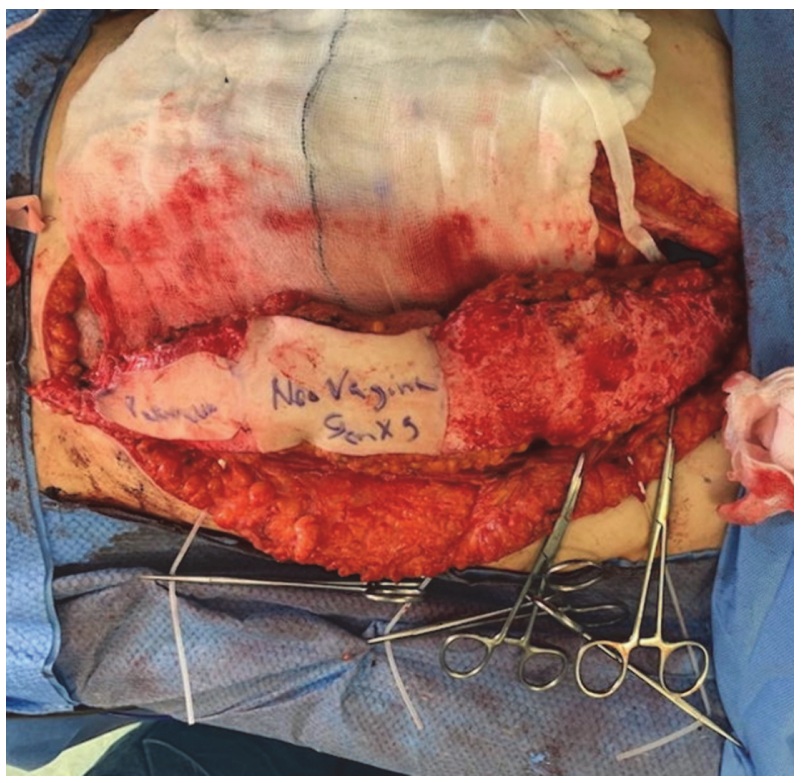

Abstract EPV262/\#462 Figure 1 